\title{
A Search for Lensing of CMB in Correlation with Large-Scale Structure
}

\author{
Christopher M. Hirata, for the SDSS collaboration
}

Department of Physics, Jadwin Hall, Princeton University, Princeton NJ 08544, USA

\begin{abstract}
We present the results of a search for gravitational lensing of the cosmic microwave background (CMB) in cross-correlation with the projected density of luminous red galaxies (LRGs). The CMB lensing reconstruction is performed using the first year of Wilkinson Microwave Anisotropy Probe (WMAP) data, and the galaxy maps are obtained using the Sloan Digital Sky Survey (SDSS) imaging data. We find no detection of lensing; our constraint on the galaxy bias derived from the galaxy-convergence cross-spectrum is $b_{g}=1.81 \pm 1.92(1 \sigma$, statistical), as compared to the expected result of $b_{g} \sim 1.7$ for this sample. We discuss possible instrument-related systematic errors and show that the Galactic foregrounds are not important. We do not find any evidence for point source or thermal Sunyaev-Zel'dovich effect contamination.
\end{abstract}

\section{Introduction}

In recent years, observations of CMB anisotropies have had enormous impact due to the robustness of the underlying theory, the rapid progress in statistical power, and the attention by the observers to minimization of systematic error. Thus far, the most important constraints have come from linear regime primary anisotropies. To the extent that the primordial perturbations are Gaussian and of scalar symmetry, these anisotropies are fully described by three power spectra $C_{\ell}^{T T}, C_{\ell}^{T E}$, and $C_{\ell}^{E E}$, and (with the exception of the integrated Sachs-Wolfe or ISW effect) are essentially independent of low-redshift observations such as galaxy surveys. But the CMB should also contain secondary anisotropies such as thermal or kinetic Sunyaev-Zel'dovich (SZ) effects, patchy reionization and gravitational lensing. These effects result from nonlinear evolution at low redshifts $z \ll 1100$. Therefore they are non-Gaussian, and there is nontrivial information in their higher-order moments and their cross-spectra with low-redshift tracers of large scale structure (LSS). Gravitational lensing of the CMB is an interesting secondary anisotropy because robust theoretical predictions of the effect are possible. Precision cosmological constraints from lensing of CMB may someday come from high-resolution temperature or, ultimately, $B$ mode polarization data. This is for the future, however present and near-term data such as that from WMAP provide us an opportunity to develop the data analysis techniques for CMB lensing, search for the effect as a "proof-of-principle" effort, and determine what real-world problems arise in the analysis. This proceeding describes an attempt to measure the cross-correlation between the density of galaxies measured in SDSS and the lensing deflection acting on the WMAP CMB observations. Here we will focus on certain aspects of the methodology and the implications for future studies; full details can be found in Hirata et al. (2004). The WMAP data set is described in Bennett et al. (2003a).

\section{Large scale structure sample}

Lensing of CMB can be studied either in autocorrelation (measuring the convergence power spectrum) or in cross-correlation with LSS. Since theoretical studies had shown that lensing maps reconstructed from WMAP were predicted to have low signal-to-noise 
ratio, we selected the latter route for our investigation. The ideal LSS sample for crosscorrelation with CMB lensing would be mapped at high signal-to-noise over a large solid angle, and probe cosmologically interesting redshifts (of order unity). The SDSS photometric luminous red galaxy (LRG) sample performs very well on the first two criteria and acceptably well on the third: the LRG clustering signal dominates over Poisson noise at $\ell \leqslant 300$, the available solid angle at the time the analysis began (fall 2003) was $\sim 3900 \mathrm{deg}^{2}$, and the redshift distribution peaks at $z \approx 0.5$. The selection of the LRGs and their redshift distribution are described in Padmanabhan et al. (2004). Details of the SDSS can be found in Fukugita et al. (1996), Gunn et al. (1998), York et al. (2000), Hogg et al. (2001), Eisenstein et al. (2001), Smith et al. (2002), Pier et al. (2003), and Blanton et al. (2003). The public SDSS data are described by Stoughton et al. (2002), Abazajian et al. (2003), Abazajian et al. (2004), and Finkbeiner et al. (2004).

\section{CMB lensing reconstruction}

CMB lensing reconstruction aims to take a map of the CMB, consisting of temperatures $\hat{T}_{i}$ (and possibly polarizations $\hat{Q}_{i}$ and $\hat{U}_{i}$ ) in each map pixel $i$, and return an estimated map $\hat{\kappa}(\hat{\mathbf{n}})$ of the convergence field. In this analysis we have used only the temperature data from WMAP, as this is publicly available and at present has much higher signal-tonoise ratio. We use the quadratic estimation method of $\mathrm{Hu}$ (2001) and Okamoto and $\mathrm{Hu}$ (2003). The basic theoretical result underlying the reconstruction methods is that the covariance of the CMB temperature Fourier modes $\dagger$ is

$$
\left\langle\hat{T}_{\vec{\ell}_{1}} \hat{T}_{\vec{\ell}_{2}}\right\rangle=C_{\ell_{1}} \delta_{\vec{\ell}_{1},-\vec{\ell}_{2}}-2\left(\vec{\ell}_{1}+\vec{\ell}_{2}\right)^{-2}\left(\vec{\ell}_{1}+\vec{\ell}_{2}\right) \cdot\left(\vec{\ell}_{1} C_{\ell_{1}}+\vec{\ell}_{2} C_{\ell_{2}}\right) \kappa_{\vec{\ell}_{1}+\vec{\ell}_{2}}+N_{\vec{\ell}_{1} \vec{\ell}_{2}}
$$

where $N_{\vec{\ell}_{1} \vec{\ell}_{2}}$ is the noise covariance and convergence $\kappa$. For fixed $\kappa$, the temperature field is Gaussian with this covariance, and so the usual quadratic estimation methods for power spectra can be applied to recover $\kappa$. In the simplest case, where the noise covariance is diagonal in Fourier space, we construct the estimator

$$
\hat{\kappa}_{\vec{\ell}}=i \ell^{-1} R_{\ell} \vec{\ell} \cdot i \sum_{\vec{\ell}_{1}+\vec{\ell}_{2}=\vec{\ell}} \vec{\ell}_{2} C_{\ell_{1}} W_{\ell_{1}} W_{\ell_{2}} \hat{T}_{\vec{\ell}_{1}} \hat{T}_{\vec{\ell}_{2}} .
$$

Here $R_{\ell}$ is chosen to make the estimator unbiased, and $W_{\ell}$ is a weight function; the optimal choice is the " $C^{-1}$ weight" $W_{\ell}=\left(C_{\ell}+N_{\ell}\right)^{-1}$. In real life, Eq. (3.2) cannot be used in this form. The WMAP noise matrix $N_{\vec{\ell}_{1} \vec{\ell}_{2}}$ is not diagonal in Fourier space, so it is necessary to subtract a "noise bias" term from Eq. (3.2), just as one does for the $\mathrm{CMB}$ power spectrum. However, a more robust way to eliminate the noise bias is to measure the two temperature modes $\hat{T}_{\vec{\ell}_{1}}$ and $\hat{T}_{\vec{\ell}_{2}}$ from different differencing assemblies (DAs) on WMAP, and then average the reconstructed map over all pairs of DAs. This approach closely parallels the WMAP power spectrum analysis of Hinshaw et al. (2003). We only use the eight $\mathrm{Q}, \mathrm{V}$, and $\mathrm{W}$ band DAs, which provide 28 quadratic estimators for $\kappa$. Another problem relates to the boundary conditions: Eq. (3.2) applies to the idealized case of periodic boundary conditions, whereas for real CMB observations the "boundaries" are the edges of the mask, e.g. the Galactic Plane cut. It is essential to construct a local lensing estimator so that artifacts from the boundaries are not smeared across the reconstructed $\hat{\kappa}$ map; we thus compute the vector field,

$$
\mathbf{v}_{\vec{\ell}}=i e^{-\ell(\ell+1) \sigma_{0}^{2} / 2} \sum_{\vec{\ell}_{1}+\vec{\ell}_{2}=\vec{\ell}} \vec{\ell}_{2} C_{\ell_{1}} W_{\ell_{1}} W_{\ell_{2}} \hat{T}_{\vec{\ell}_{1}} \hat{T}_{\vec{\ell}_{2}} .
$$

$\dagger$ In these proceedings we will show only the flat-sky expressions for simplicity; the actual analysis uses the full-sky expressions, which can be found in Hirata et al. (2004). 
Galaxy-convergence correlation
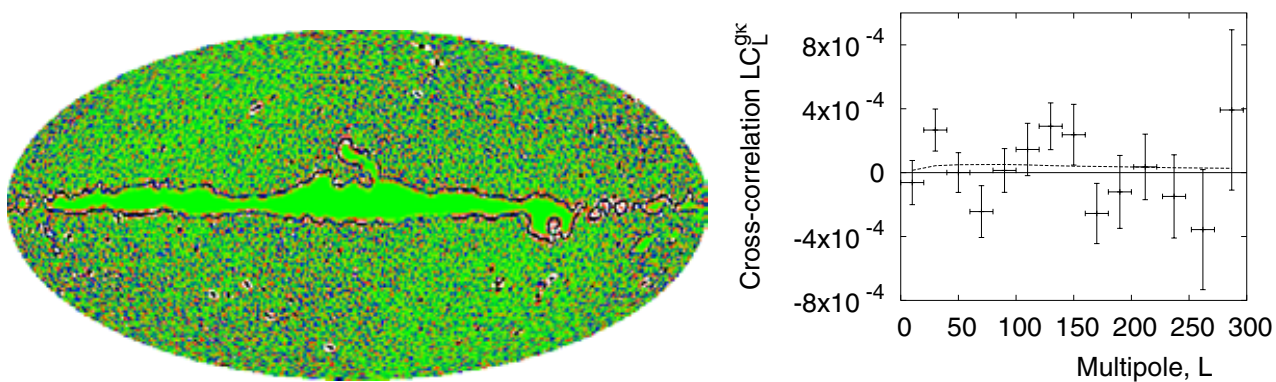

Figure 1. Left: The lensing map $\nabla \cdot \mathbf{v}$, smoothed with a 30 arcmin FWHM Gaussian, in Galactic Molleweide projection. Note the artifacts around point sources and the Galactic Plane cut, which were masked for the analysis. Right: The galaxy-convergence cross spectrum $C_{\ell}^{g \kappa}$, measured from SDSS LRGs and WMAP first-year CMB maps. The dashed line is the best-fit signal.

If $W_{\ell}$ and $C_{\ell}$ have smooth $\ell$-dependence so that the convolved functions $W \hat{T}$ and $C W \hat{T}$ are local functions of the data, it follows that $\mathbf{v}$ is also a local function of the data (since $i \overrightarrow{\ell_{2}}$ is simply a gradient acting on $W \hat{T}$ ). Once the galaxy-v cross-spectrum is measured, it is easy to recover the galaxy-convergence cross-spectrum by inserting a factor of $R_{\ell}$. There is a difficulty when we wish to cross-correlate $\mathbf{v}$ with another field (e.g. the LRG density map) using a "pseudo- $C_{\ell}$ " type estimator since the extremely short-wavelength modes of $\mathbf{v}$ contain lots of power and boost the noise in the $C_{\ell}^{g \mathbf{v}}$ estimator for low $\ell$; we eliminate this problem by inserting the Gaussian smoothing $e^{-\ell(\ell+1) \sigma_{0}^{2} / 2}$ with $\sigma_{0}=34$ arcmin.

We also found that the lensing estimator was being contaminated by ringing artifacts from the Galactic Plane. We solved this problem by masking the worst part of the Galactic Plane [Kp4; Bennett et al. (2003b)] first, then applying the lensing reconstruction algorithms; artifacts are observed right at the edge of the Galactic Plane, and these are then masked. A final problem with Eq. (3.2) is point sources. At $41 \mathrm{GHz}$, we found that radio point sources dominate the power spectrum of the reconstructed lensing map $\mathbf{v}$ at $\ell<30$ and are a significant contaminant at all multipoles; unsurprisingly, the contamination is dramatically reduced at higher frequencies. Our solution in this analysis was to reject a 2 degree radius "contaminated region" around each source. This results in the loss of $21 \%$ of the usable area of the CMB lensing map; while this is acceptable for this analysis it will eventually need to be improved, particularly as CMB experiments measure smaller-scale temperature modes for which the point sources are more important. The lensing sky map is shown in the left panel of Fig. 1 (almost all the power in the map is "shape noise" from the CMB spots or detector noise). Simulations show the resulting estimator for $C_{\ell}^{g \kappa}$ to be calibrated to within $18 \pm 7 \%$ ( $1 \sigma$, Monte Carlo error).

\section{Results and foregrounds}

Having obtained the galaxy map $g=\delta n / \bar{n}$ and the lensing map $\mathbf{v}$, we proceeded to compute the cross-spectrum $C_{\ell}^{g \kappa}$, shown in the right panel of Fig. 1 . The best-fit galaxy bias is $b_{g}=1.81 \pm 1.92(1 \sigma)$. This is consistent with other results - Padmanabhan et al. (2004) find $b_{g} \sim 1.7$ from the LRG autopower spectrum - but does not yield a detection.

CMB lensing measurements can in principle be contaminated by foreground microwave emission of Galactic and extragalactic origin. The simplest way to model the effects of Galactic foregrounds on the CMB lensing analysis is to feed foreground maps through the analysis pipeline in place of the real CMB maps, and calculate the resulting contamination 
to the galaxy-convergence correlation and the galaxy bias; the spurious contribution due to the foregrounds is estimated to be $\Delta b_{g}=-4.5 \times 10^{-4}$, i.e. it is negligible.

The extragalactic foregrounds are harder to constrain. If we assume that the point sources have the $I_{\nu}=$ constant spectrum typical of synchrotron radiation, then we can search for a frequency dependence of the lensing signal. Since the lensing estimator is a quadratic function of the CMB temperature, we have six frequency combinations available: QQ, QV, QW, VV, VW, and WW. Fitting a combination of blackbody (CMB) + point sources to the computed bias signal yields a frequency-averaged point source contamination of $\Delta b_{g}^{(P S)}=0.73 \pm 1.18$; thus while there is clearly no detection of point sources, the frequency information alone does not establish the point sources as negligible. See Hirata et al. (2004) for tighter constraints on point sources including assumptions about the spatial dependence. A final concern is thermal SZ; we have not examined this contaminant in detail since we do not detect any signal (although our constraint on $b_{g}$ shows that the SZ does not exceed the lensing signal by orders of magnitude). Multifrequency information will of course be needed for CMB lensing studies at high $\ell$ where thermal SZ dominates over primary temperature fluctuations.

\section{Conclusion: future prospects}

We have argued that the correlation of LSS with lensing of CMB is a potentially very robust cosmological, but as yet the statistical power is lacking. The signal-to-noise ratio for the cross-correlation $C_{\ell}^{X \kappa}$ between CMB lensing and tracer $X$ of LSS is given by

$$
\frac{d(S / N)^{2}}{d \ln \ell}=\frac{\ell^{2}}{2 \pi} \Omega \frac{\rho_{\ell}^{2}}{1+\rho_{\ell}^{2}+N_{\ell}^{\kappa \kappa} / C_{\ell}^{\kappa \kappa}},
$$

where $\rho_{\ell}$ is the correlation coefficient between $X$ and $\kappa, \Omega$ is the observed solid angle, and $N_{\ell}^{\kappa \kappa}$ is the noise power spectrum of the reconstructed convergence. At present, $N_{\ell}^{\kappa \kappa} \gg C_{\ell}^{\kappa \kappa}$ so that the noise term in Eq. (5.1) dominates the denominator. It is apparent that achieving high signal-to-noise will require measuring many lensing modes $\ell^{2} \Omega$, selecting tracers with high correlation coefficient $\rho_{\ell}$, and/or reducing the CMB lensing reconstruction noise $N_{\ell}^{\kappa \kappa} / C_{\ell}^{\kappa \kappa}$. The reconstruction noise is plotted in Fig. 2a for WMAP, Planck $\dagger$, and a possible future polarization-sensitive satellite.

In Fig. 2b, we have shown the correlation coefficients for several tracers of LSS including LRGs, cosmic shear, and ISW. The LRGs have a moderate $\geqslant 0.2$ correlation coefficient on large scales have already been mapped over $3900 \mathrm{deg}^{2}$ (which will increase by an additional several thousand $\operatorname{deg}^{2}$ as the SDSS progresses), hence they are a good candidate for "first detection" studies. Ultimately however, precision cosmology may demand a cross-correlation with "cleaner" theory than the LRGs. The ISW-lensing correlation can directly probe deviations from an $\Omega=1$ universe [Seljak and Zaldarriaga (1999)], however as we can see from the figure the signal is limited to low $\ell$ and hence cosmic variance limits the available $S / N$ (cf. the factor of $\ell^{2}$ in Eq. 5.1). Wide-angle cosmic shear surveysł will provide high $\rho_{\ell}$ over a range of scales; the curve in Fig. 2 shows predictions for a survey with median redshift $z_{s}=1$ and 30 galaxies/arcmin ${ }^{2}$. This type of crosscorrelation is potentially more robust against sources of spurious power than the cosmic shear derived from galaxies or CMB alone, although it is still subject to contamination from intrinsic alignments [Hirata and Seljak (2004)].

$\dagger$ http://www.rssd.esa.int/index.php?project=PLANCK

$\ddagger$ See, e.g. Pan-STARRS (http://pan-starrs.ifa.hawaii.edu/public/); SNAP (http://snap.lbl.gov/); and LSST (http://www.lsst.org/). 
(a) Lensing reconstruction noise

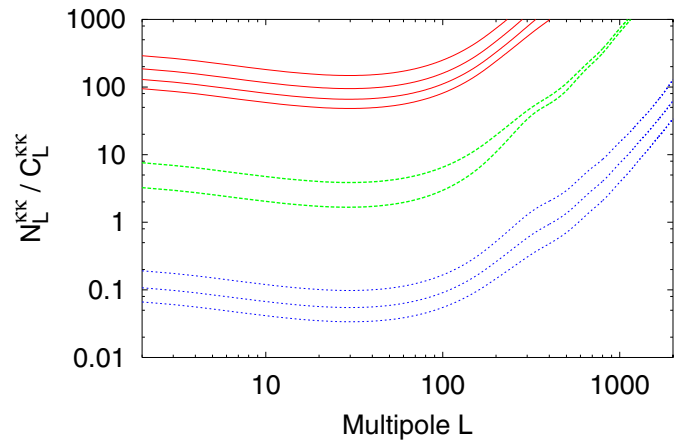

(b) Correlation coefficient with LSS tracers

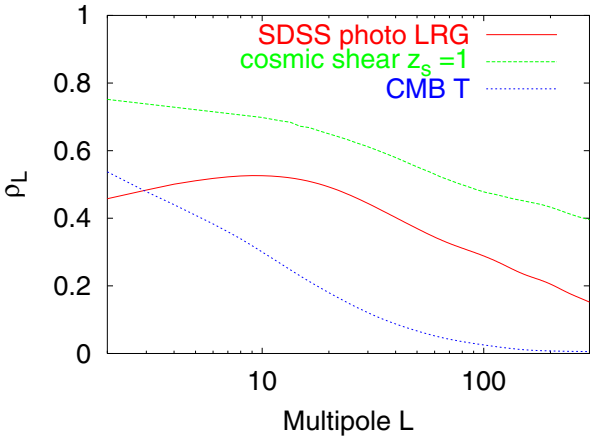

Figure 2. (a) The lensing reconstruction noise for various experiments as a function of $\ell$. The top (thin solid) group of curves is for WMAP 1,2, 4, and 8 year data (top to bottom). The middle pair of curves is for Planck 14 month data with the $217 \mathrm{GHz}$ temperature channel (upper curve) and also including 100, 143, and $217 \mathrm{GHz}$ polarization (lower curve). The bottom triplet of curves is for a future polarization-sensitive satellite with noise of 10,5 , and $2.5 \mu \mathrm{K}$ arcmin on each Stokes parameter $(Q$ or $U)$ and a 5 arcmin beam, using quadratic reconstruction. (b) The correlation coefficient $\rho_{\ell}$ for three tracers of LSS: the SDSS LRGs, cosmic shear, and ISW effect. The calculations include the suppression of $\rho_{\ell}$ due to Poisson noise, shape noise, and primary CMB respectively, which adds uncorrelated power to the LSS map.

Ultimately, a CMB polarization satellite and an overlapping cosmic shear yield $S / N$ on the cross-spectrum of $\sim 100 f_{s k y}^{1 / 2}$. The CMB polarization data would yield a sampling variance-limited convergence map out to $\ell \sim$ few $\times 10^{2}$, thereby enabling us to probe the matter power spectrum at very large scales [Hu and Okamoto (2002)]. But we must not minimize the challenges. In order to realize the full potential of CMB lensing, we will need detectors with several $\mu \mathrm{K}$ arcmin sensitivity over large portions of the sky, with commensurate control of systematics. Extragalactic foregrounds, particularly kinetic SZ and patchy reionization that share the frequency dependence of the CMB, will also be important on small scales [Amblard et al. (2004)]. The level of polarized foregrounds remains uncertain, although the next generation of $E$-mode experiments will provide important data on these. Finally, the statistical and computational aspects of CMB are a rich area for investigation. While much attention has been devoted to Gaussian power spectrum estimation, lensing analyses examine higher-order moments of the CMB and LSS [Kesden et al. (2003)], and Hirata and Seljak (2003) showed in simulations of CMB polarization that "iterative" estimators that are non-polynomial functions of $Q$ and $U$ can reconstruct the lensing field better than quadratic estimators. Constructing fast lensing estimators that deal with elliptical beams, point source cuts, survey boundaries, etc. will be essential, but to date little work has been done in these non-idealized situations or on speeding up algorithms for CMB lensing. If these challenges can be met, the lensing of the CMB will provide us with a powerful and robust probe of the low-redshift universe.

\section{Acknowledgements}

I thank my collaborators on this project: N. Padmanabhan, U. Seljak, D. Schlegel, and J. Brinkmann. We acknowledge useful discussions with N. Afshordi, J. Hennawi, Y.-S. Loh, and L. Page. C.H. is supported by NASA NGT5-50383. Some of the results in this paper have been derived using the HEALPıx [Górski and et al. (1999)] package. We acknowledge the use of the Legacy Archive for Microwave Background Data Analysis (LAMBDA). Support for LAMBDA is provided by the NASA Office of Space Science. 
Funding for the creation and distribution of the SDSS Archive has been provided by the Alfred P. Sloan Foundation, the Participating Institutions, the National Aeronautics and Space Administration, the National Science Foundation, the U.S. Department of Energy, the Japanese Monbukagakusho, and the Max Planck Society. The SDSS Web site is http://www.sdss.org/. The SDSS is managed by the Astrophysical Research Consortium (ARC) for the Participating Institutions. The Participating Institutions are The University of Chicago, Fermilab, the Institute for Advanced Study, the Japan Participation Group, The Johns Hopkins University, Los Alamos National Laboratory, the Max-Planck-Institute for Astronomy (MPIA), the Max-Planck-Institute for Astrophysics (MPA), New Mexico State University, University of Pittsburgh, Princeton University, the United States Naval Observatory, and the University of Washington.

\section{References}

K. Abazajian et al., Astron. J. 126, 2081-2086 (2003).

K. Abazajian et al., Astron. J. 128, 502-512 (2004).

A. Amblard, C. Vale, and M. White, astro-ph/0403075 (2004).

C. L. Bennett et al., Astrophys. J. Supp. 148, 1-27 (2003a).

C. L. Bennett et al., Astrophys. J. Supp. 148, 97-117 (2003b).

M. R. Blanton et al., Astron. J. 125, 2276-2286 (2003).

D. J. Eisenstein et al., Astron. J. 122, 2267-2280 (2001).

D. Finkbeiner et al., Astron. J., submitted. (2004).

M. Fukugita et al., Astron. J. 111, 1748-1756 (1996).

K. M. Górski et al., in Evolution of Large Scale Structure : From Recombination to Garching (1999), pp. 37-42.

J. E. Gunn et al., Astron. J. 116, 3040-3081 (1998).

G. Hinshaw et al., Astrophys. J. Supp. 148, 135-159 (2003).

C. M. Hirata and U. Seljak, Phys. Rev. D 68, 83002 (2003).

C. M. Hirata and U. Seljak, Phys. Rev. D, accepted, astro-ph/0406275 (2004).

C. M. Hirata, N. Padmanabhan, U. Seljak, D. Schlegel, and J. Brinkmann, Phys. Rev. D, submitted, astro-ph/0406004 (2004).

D. W. Hogg, D. P. Finkbeiner, D. J. Schlegel, and J. E. Gunn, Astron. J. 122, 2129-2138 (2001).

W. Hu and T. Okamoto, Astrophys. J. 574, 566-574 (2002).

W. Hu, Astrophys. J. 557, L79-L82 (2001).

M. Kesden, A. Cooray, and M. Kamionkowski, Phys. Rev. D 67, 123507 (2003).

T. Okamoto and W. Hu, Phys. Rev. D 67, 83002 (2003).

N. Padmanabhan et al., Mon. Not. R. Astron. Soc., submitted, astro-ph/0407594, (2004).

J. R. Pier et al., Astron. J. 125, 1559-1579 (2003).

U. Seljak and M. Zaldarriaga, Phys. Rev. D 60, 43504 (1999).

J. A. Smith et al., Astron. J. 123, 2121-2144 (2002).

C. Stoughton et al., Astron. J. 123, 485-548 (2002).

D. G. York et al., Astron. J. 120, 1579-1587 (2000).

\section{Discussion}

SARAH BRIDLE: Do you need to assume anything about the mass-to-light ratio for the LRGs in this method?

Christopher Hirata: No, we only use the number counts for the LRGs, not their luminosities. The assumption here is that the LRGs are related to the density field via a linear bias relationship, $\delta_{L R G}=b_{g} \delta_{m}$, and the objective is to measure $b_{g}$. For a fixed cosmology, the observable cross-spectrum $C_{\ell}^{g \mathbf{v}} \propto b_{g}$ and so we do a fit to find $b_{g}$. 